1 Smell-based memory training: Evidence of olfactory learning and transfer to

2 the visual domain

3

4 Authors: Jonas K. Olofsson ${ }^{1-2 *}$, Ingrid Ekström ${ }^{1 *}$, Joanna Lindström ${ }^{1}$, Elmeri Syrjänen ${ }^{1}$, Anna

5 Stigsdotter-Neely ${ }^{3}$, Lars Nyberg ${ }^{4-6}$, Sara Jonsson $^{1}$, and Maria Larsson ${ }^{1}$.

$7 \quad$ *The authors contributed equally to this work

8 Affiliations: ${ }^{1}$ Gösta Ekman Laboratory, Department of Psychology, Stockholm University,

9 Stockholm, Sweden; ${ }^{2}$ Swedish Collegium of Advanced Study, Uppsala, Sweden; ${ }^{3}$ Department

10 of Social and Psychological Studies, Karlstad University, Karlstad, Sweden; ${ }^{4}$ Umeå Center for

11 Functional Brain Imaging, Umeå University, Umeå, Sweden; ${ }^{5}$ Department of Radiation

12 Sciences, Umeå University, Umeå, Sweden; ${ }^{6}$ Department of Integrative Medical Biology, Umeå

13 University, Umeå, Sweden.

15 Author contributions: The study concept was developed by J.K.O. All authors contributed to

16 the study design. Data collection and analysis was conducted by authors I.E., J.L, S.J., and E.S.,

17 under the supervision of J.K.O and M.L. Authors J.K.O and I.E drafted the manuscript, which

18 was revised based on input from all other authors. All authors approved of the final version of

19 the manuscript.

21 Corresponding author: Jonas K. Olofsson. Email: Jonas.olofsson@psychology.su.se; tel: +46-

22 8-163840; fax: +46-8-162000.

23 Word count: 4774 (intro, methods, results, discussion, tables figures and legends) 


\section{Smell-based memory training: Evidence of olfactory learning and transfer to}

\section{5 the visual domain}

26 Abstract: Human and non-human animal research converge to suggest that the sense of smell,

27 olfaction, has a high level of plasticity and is intimately associated with visual-spatial orientation

28 and memory encoding networks. We investigated whether olfactory memory training would lead

29 to transfer to an untrained visual memory task, as well as untrained olfactory tasks. We devised a

30 memory intervention to compare transfer effects generated by olfactory and non-olfactory

31 (visual) memory training. Adult participants were randomly assigned to daily memory training

32 for about 40 days with either olfactory or visual tasks, that had a similar difficulty level. Results

33 showed that while visual training did not produce transfer to the olfactory memory task,

34 olfactory training produced transfer to the untrained visual memory task. Olfactory training also

35 improved participants' performance on odor discrimination and naming tasks, such that they

36 reached the same performance level as a high-performing group of wine professionals. Our

37 results indicate that the olfactory system is highly responsive to training, and we speculate that

38 the sense of smell may facilitate transfer of learning to other sensory domains. Further research is

39 however needed in order to replicate and extend our findings.

41 Public Significance Statement: This study suggests that olfaction, the sense of smell, might be

42 helpful in memory training, as olfactory-based memory training benefitted visual memory task

43 performance. Olfactory-based training also led to improvement in some olfactory abilities, such

44 that novices, following training, achieved the same level of performance as wine professionals.

46 Keywords: Smell, olfactory disorders, odorants, memory, spatial learning 


\section{Introduction}

48 Olfaction, the sense of smell, is highly associated with learning, and some studies suggest that

49 olfactory sensory abilities even play a role in visual memory performance. For example, odor

50 discrimination training leads to visuo-spatial learning enhancement in rats (Zelcer et al., 2006).

51 Conversely, an ablation of the olfactory bulb impairs visuo-spatial learning in rats (van Rijzingen

52 et al., 1995). The present study focuses on whether olfactory-based memory training in adult

53 human participants would have positive effects both on visual memory and olfactory perceptual

54 task performance. The olfactory system is characterized by a high level of biological and functional

55 plasticity (Fletcher, 2012; Kass et al., 2013; Li et al., 2008). For example, healthy adult humans

56 might improve olfactory performance merely by means of repeated olfactory stimulation

57 (Mainland et al., 2002), and to our knowledge, no analogous effects have been reported in the

58 visual system. Olfactory abilities are highly trainable (Damm et al., 2014; Hummel et al., 2009;

59 Morquecho-Campos et al., 2019). Olfactory experts (e.g. perfumers and wine tasters) perform

60 better than non-experts on olfactory assessments (Royet et al., 2013) and their expertise is

61 correlated with structural reorganizations in memory-associated brain areas (Delon-Martin et al.,

62 2013). A preliminary study, while lacking a matched control group, indicated that odor-based

63 spatial memory training might benefit performance on related visual cognitive tasks (Olofsson et

64 al., 2017). Memory transfer is often studied using visual, and in some cases auditory tasks, and

65 results indicate that while some studies have yielded promising results (e.g. Mahncke et al., 2006;

66 Bergman-Nutley \& Klingberg 2014), others show no transfer (Owen et al., 2010), or only to tasks

67 that are very similar to the trained task, such that the overall pattern of results remain mixed

68 (Lindenberger et al., 2017; Melby-Lervåg et al., 2016; Teixeira-Santos et al., 2019). Transfer

69 across sensory systems are rarely assessed (see e.g. Zelinski, 2009, for review). Previous studies 
70 investigated whether visual working memory training transfers to improved performance on a

71 similar auditory working memory task, but results are not conclusive (Buschkuehl et al., 2014;

72 Schneiders et al., 2011). As the usefulness of some visual-based cognitive training interventions

73 has been questioned on methodological grounds (Simons et al., 2016), new methods for

74 establishing transfer of learning are warranted. To our knowledge, differences in cross-sensory

75 transfer has never been systematically addressed using olfaction. Olfaction, with its combination

76 of high levels of plasticity and its close integration with memory encoding regions, might provide

77 a vehicle for transfer effects. This study aimed to test the hypothesis, based on the evidence

78 reviewed above (pronounced olfactory plasticity and transfer effects), that memory training in the

79 olfactory system would lead to significant transfer to a visual task, as well as to non-trained

80 olfactory tasks. We further hypothesized, based on the indirect evidence reviewed above (modest

81 transfer within the visual cognitive domain and lack of evidence of visual transfer to olfactory

82 tasks) that transfer from visual training to olfactory tasks would be less pronounced. To address

83 this hypothesis, we devised two spatial learning board games where olfactory and visual objects

84 were located and matched. Such tasks are known to engage memory encoding networks (Kunz et

85 al., 2015; Rasch et al., 2007) and we used them to establish training-related gain and transfer.

\section{$87 \quad$ Materials and methods}

88 Participants

89 This study was approved by the Swedish Ethics Review Authority (2014/621-31/1) and complied

90 with the Declaration of Helsinki for Medical Research involving Human Subjects. Participants

91 ( $\mathrm{n}=106$; age 18-50 years) were recruited to olfactory and visual memory training conditions by

92 means of advertising, mainly through a designated website for research study advertisements. 
93 Exclusion criteria were neurologic or psychiatric disorders, colds, allergies or breathing

94 problems, and olfactory or uncorrected visual impairments. Participants were randomly assigned

95 to either olfactory memory (OM) or visual memory (VM) training groups, and were tested by

96 trained research assistants who were blind to the participants' training. Participants were

97 compensated monetarily after completion of the training program. Seventy-two participants

98 completed training, pre-test and post-test; 41 with the olfactory memory task (OM) and 31 with

99 the visual memory task (VM), The effective sample is higher than, or similar to, several other

100 studies on this topic, which should provide a sufficient power to detect effects (e.g. Hummel et

101 al., 2009; Altundag et al., 2015). Twenty-eight participants did not complete memory training or

102 deviated from the training protocol (17 OM, $11 \mathrm{VM}$; the OM training was slightly more

103 vulnerable to disruptions from cold and flu). Additionally, 6 participants were excluded due to

104 technical or experimenter errors during initial testing. The current dataset with 72 participants

105 includes participants which were recruited to a study that was originally conceived as a follow-

106 up, where participants were randomized to identical olfactory and visual training conditions and

107 identical pre- and post-test tasks. The second study, which also included two other training

108 conditions and a novel set of criterion tasks, was scaled down midway through data collection for

109 practical reasons, starting with the visual condition. The data from the follow-up study (11 VM,

$11020 \mathrm{OM}$ ) were instead aggregated with the original study data to achieve more reliable outcomes,

111 which explains the lower number of participants in the visual condition (see online supplemental

112 materials for data). Post-hoc exploration indicated that original vs. follow-up datasets showed

113 similar results.

114 


\section{Wine professionals}

117 To provide a point of reference for the hypothesized training-related olfactory improvement in the

118 OM group, we additionally recruited 15 wine professionals for testing with the Sniffin' Sticks

119 battery. They were recruited through a professional network email list and were currently active

120 as wine panelists, wine tasting course leaders, wine importers or wine journalists (see

121 supplementary materials for descriptive and performance data). Even though the professionals had

122 a higher mean age than particpants in the training groups, we decided it was more important to

123 have professionals with many years of experience as a bench-mark for olfactory expert

124 performance (Delon-Martin et al., 2013); rather than striving for an age-matched group of young,

125 but less experienced, olfactory experts (e.g. enology students).

\section{Training tasks}

128 The olfactory memory game (OM) included 24 tin cans, containing 12 different kinds of 129 commercially available flavored teas to make up 12 stimulus pairs (see supplementary materials).

130 Two parallel, non-overlapping sets were created to make the olfactory and visual games more 131 challenging and to involve a larger number of stimuli during the training period, a feature we hoped

132 would help us achieve generalized learning and avoid minimize unknown sources of bias driven

133 by individual stimuli. Prior work in patients with olfactory loss has shown better results from 134 olfactory training when odors are changed during training (Altundag et al., 2015). Odors were 135 placed in white cotton bags to prevent visual identification. The cans were randomly distributed 136 on a board that included 24 squares arranged in a 6-by-4 grid. On each trial, the player sampled 137 two tin cans of choice and compared their content. Upon detecting a match, the matched items 138 were removed from the board. Performance was measured as the number of trials required to empty 
139 the board, with fewer trials indicating higher performance. Trial number was also used to compare

140 the difficulty of the two tasks. In order to establish accurate performance logs, each tin can was

141 marked underneath with a barcode that was unique to each can, but mirror-inverted for the can

142 holding the matching stimulus (see supplementary materials). Thus, participants could easily

143 verify a match by turning two cans upside down and holding the barcodes opposite each other.

144 However, the mirror-reversed barcodes were not visible while playing, and participants were

145 instructed to only use them to confirm a perceived match, limiting exposure to the barcodes mostly

146 to trials where the cans were immediately removed from the board. Barcodes were thus unlikely

147 to influence memory performance, since they could not be used as a memory cue for the matching

148 task (see supplementary materials).

150 A visual memory (VM) game was devised as a control task. Two parallel, non-overlapping sets

151 were constructed. In each set, 12 different language symbols were obtained from Korean and 152 Mandarin Chinese languages, and placed inside the 24 tin cans (see supplementary materials). The 153 two training tasks were similar in all aspects except the engagement of odors vs. visual objects and 154 they were carried out in a similar way. The symbols were unfamiliar to our Swedish participants.

156 Procedure

157 Completion of the study required training for about 40 days at home (one training session 158 completed each day, average training time was 10 minutes) and pre-training and post-training 159 laboratory assessment. In cases when participants were unable to complete training on a given 160 day, they were instructed to train twice the following day(s) such that their total number of 161 training sessions were kept constant. Post-training assessments were scheduled after at least 38 
162 days of training and at about 40 days (see Table 1 for details), and participants continued training

163 until the day of return. During the laboratory assessments, participants completed tests with the

164 OM and VM tasks as well tests of olfactory function. During training, participants trained with

165 only one type of game, but alternated between the parallel sets every 5 days.

166

167 The games were played individually. Participants were instructed to place all the cans on the 168 game board in a random order and play the game by sampling two cans on each trial, removing 169 the cans if they were matching. Each trial was documented on a scoring sheet as "matching" or

170 "non-matching". After a minimum of 38 training days, participants returned for the second

171 laboratory assessment. Participants that could not return at 38-40 days from their pre-assessment

172 were either instructed to continue training for a few more days until they were available for

173 laboratory testing or, if this was not possible, considered as drop-outs.

174

175 Pre-post testing. Pre-training and post-training sessions followed the same procedure and

176 consisted of standardized tests of olfactory ability and assessments of the participants

177 performance in the olfactory and visual memory games. First, the standardized "Sniffin' Sticks"

178 were used to assess olfactory abilities (see Hummel et al., 1997, 2007; Kobal et al., 1996, for

179 details). Olfactory threshold (for n-butanol) and discrimination assessments were completed in

180 exact accordance to established protocols, and odor sets were replaced within their expiry date.

181 In threshold testing, the experimenter presents the participant with three odorous pens, only one

182 of which contains an odor (n-butanol), and the participant selects the odorous pen in a forced-

183 choice procedure. Across trials, the concentration of the odor stimulus varied as a function of

184 performance, and a threshold value of up to 16 was eventually established. In discrimination 
185 testing, three pens are similarly presented, two of which has the same smell and the third has a 186 different smell, and the participant selects the deviating smell in a forced choice procedure.

187 Discrimination ability is established as a sum score across 16 trials. For naming and

188 identification assessments, participants were presented with familiar odors, and were first asked

189 to name it without cues; these answers provided the basis for calculating odor naming accuracy

190 scores (naming is not part of the standard Sniffin' Sticks assessment, but is often added to

191 identification assessments to provide complementary information, see e.g. Olofsson et al., 2013).

192 Irrespective of the naming response, odor identification was then assessed by providing four odor 193 name alternatives (one of which was correct) in a forced-choice task. To avoid test-retest effects

194 on the olfactory identification and naming tests, we used an extended version of the Sniffin'

195 Sticks set that contained 32 odors, which were divided into two non-overlapping sets of 16 (8

196 odors from the original Sniffin' Sticks set and 8 odors from its extension set), for unique use at

197 test and re-test, respectively. The use of each of the two alternate sets at pre-test and post-test

198 was randomized for each individual to minimize trivial test-retest effects in the identification and

199 naming tasks. Scores on olfactory tasks could range from 0 (min) to 16 (max). Participants were

200 not given any corrective feedback. We hypothesized all four tests would potentially be improved

201 only by olfactory training, resulting in interactions between time and group.

203 Participants' performance in the training games was assessed. Assessment order (olfactory

204 memory first versus visual memory game first) was randomized across participants. The

205 experimenter instructed the participant in how to play the memory games and how to document

206 performance (number of trials) into the accompanying spreadsheet according to the same

207 procedure as training at home. After verifying that the participant had understood the instructions 
208 correctly by letting the participant play and document two test trials under supervision, the test

209 leader left the room and returned once the participant was finished with the game. After

210 completion, the test leader controlled that the participant had found and grouped all matching

211 cans correctly and that the spreadsheets were correctly documented.

212

213 Questionnaires. Before the first and the second testing session, participants filled out an online

214 questionnaire at home that assessed background variables such as level of education, age, gender,

215 and self-rated health, memory, and olfaction. At the post-training session, subjects rated their

216 motivation retrospectively for the first and last weeks of training, how demanding they perceived

217 the game to be, and how much they enjoyed playing the game, on scales ranging from 1 (min) to $21810(\max )$.

219

220 Data Analysis. To provide an assessment of ceiling-level performance in the memory games, we 221 simulated the performance of a group of 10000 "virtual experts" that explored the stimulus set 222 randomly but with optimal perception and memory of the objects encountered at all previous 223 trials such that performance was optimized. Although variance occurred as a function of random 224 localization of game items on the game board, and the random exploration of novel items, a 225 player with perfect memory would need an average of 18.9 trials $(\mathrm{SD}=0.83$; code available 226 upon request). Assessment of performance differences due to training was established using t227 tests and ANOVAs where appropriate. 
Demographics, olfactory performance and self-rated variables. Descriptive data are presented in

231 Table 1. T-tests indicated that groups were not different with regards to age $(\mathrm{t}(1,70)=0.967, p=$

232.337 , Hedges's $g=0.228)$ and education $(\mathrm{t}(1,70)=0.473, p=0.639$, Hedges's $g=0.118)$, and a

233 pearson chi-square test indicated no difference in sex distribution $(\mathrm{X} 2=0.212, p=0.625$, $\mathrm{phi}=$

234 0.054). The two groups did not differ in terms of baseline performance on any olfactory task (all

$235 p s>0.145)$. All participants were enrolled in the study for at least 38 days of training. The

236 number of days in the study varied somewhat among participants due to their availability for

237 post-training lab testing (see Table 1) but an independent t-test on all participants, except 3 for

238 which these data were lost, indicated groups did not differ in this regard $(\mathrm{t}(1,67)=0.540, p=$

2390.591 , Hedges's $g=0.125$ ). We ran ANOVAS to investigate effects of time (pre- vs. post-

240 training) on olfactory control tests and self-reported variables. Odor threshold and identification

241 scores did not differ statistically between groups or as a function of time, nor were there any

242 significant interactions (all $p s>0.145$ ). Increased performance from pre-test to post-test was

243 observed in odor naming $\left(\mathrm{F}(1,68)=5.363, p=0.023, \eta_{\mathrm{p}}{ }^{2}=0.071\right)$ and discrimination $(\mathrm{F}(1,68)=$

$\left.2444.245, p=0.043, \eta_{\mathrm{p}}{ }^{2}=0.057\right)$, but these effects were qualified by interactions between time and

245 group, both for naming $\left(\mathrm{F}(1,68)=7.835, \mathrm{p}=0.007, \eta_{\mathrm{p}}{ }^{2}=0.101\right)$ and for discrimination $(\mathrm{F}(1,68)$

$246=23.197, p<0.001, \eta_{\mathrm{p}}^{2}=0.249$ ); these interactions warranted follow-up ANOVAs targeting

247 each group. These analyses showed training-related effects were present only in the OM group

248 (discrimination $\mathrm{F}(1,40)=25.333, p<0.001, \eta_{\mathrm{p}}{ }^{2}=0.388$; naming $\mathrm{F}(1,40)=21.681, p<0.001, \eta_{\mathrm{p}}{ }^{2}$

$249=0.352$ ) but not in the visual group (discrimination $\mathrm{F}(1,30)=3.755, p=0.062, \eta_{\mathrm{p}}{ }^{2}=0.111$;

250 naming $\left.\mathrm{F}(1,30)=0.073, p=0.788, \eta_{\mathrm{p}}{ }^{2}=0.002\right)$. Self-rated motivation and enjoyment was

251 initially high in both groups, but declined to moderate levels during the training period, a decline

252 that was significant for motivation $\left(\mathrm{F}(1,70)=29.8, p<0.001, \eta_{\mathrm{p}}{ }^{2}=0.299\right)$ and enjoyment 
$253\left(\mathrm{~F}(1,70)=37.9, p<0.001, \eta_{\mathrm{p}}^{2}=0.351\right.$ ), but did not differ for the two groups (no main or 254 interaction effects involving group, all $p \mathrm{~s}<0.088$ ). Perceived difficulty of the tasks was 255 moderate, similar across the training groups and remained stable during the training period (all $256 p s>0.070)$. 
Table 1. Mean demographic, Sniffin' sticks and questionnaire data for the olfactory and visual training groups at

260 pre- and at post-test.

\begin{tabular}{|c|c|c|c|c|}
\hline & \multicolumn{2}{|c|}{ Pre-assessment } & \multicolumn{2}{|c|}{ Post-assessment } \\
\hline & Olfactory group & Visual group & Olfactory group & Visual group \\
\hline Age (+-SD) & $25.6(5.0)$ & $27.0(7.5)$ & - & - \\
\hline n (\% female) & $41(66)$ & $31(71)$ & - & - \\
\hline Education years (+-SD) & $14.3(1.7)$ & $14.6(2.7)$ & - & - \\
\hline Days until post-test (+-SD) & - & - & $42.7(5.9)$ & $43.4(4.1)$ \\
\hline Odor threshold (+-SD) & $11.2(3.3)$ & $10.8(2.8)$ & $11.6(3.3)$ & $10.8(2.8)$ \\
\hline Odor discrimination (+-SD) & $12.3(2.1)$ & $12.9(1.8)$ & $13.9(1.7)$ & $12.3(1.7)$ \\
\hline Odor identification (+-SD) & $12.52 .0)$ & $12.4(1.7)$ & $12.9(2.0)$ & $12.8(2.3)$ \\
\hline Odor naming (+-SD) & $5.4(1.9)$ & $6.2(2.8)$ & $7.1(2.4)$ & $6.0(2.4)$ \\
\hline Odor memory task (+-SD) & $38.1(10.8)$ & $40.1(10.5)$ & $28.3(7.3)$ & $38.5(9.6)$ \\
\hline Visual memory task (+-SD) & $33.8(10.3)$ & $37.9(8.8)$ & $26.5(7.2)$ & $24.4(6.7)$ \\
\hline Self-rated motivation (1-10) & $7.5(1.9)$ & $7.1(2.3)$ & $5.9(2.4)$ & $5.9(2.2)$ \\
\hline Self-rated enjoyment of task (1-10) & $7.5(1.8)$ & $7.0(1.9)$ & $5.1(2.2)$ & $5.7(2.5)$ \\
\hline Perceived difficulty of task (1-10) & $4.7(2.9)$ & $4.4(2.4)$ & $4.8(2.4)$ & $3.5(1.9)$ \\
\hline
\end{tabular}

$\mathrm{SD}=$ Standard Deviation; $\mathrm{n}=$ sample size .

263 Memory game performance. Mean performance scores in the training games for each training

264 group at pre- and post-assessment are summarized in Table 1. Participants did not differ

265 significantly in baseline performance in the olfactory $(\mathrm{t}(70)=0.76, p=0.452$; Hedges's $g=$

$2660.18)$ or visual memory game $(\mathrm{t}(70)=1.80, p=0.077$; Hedges's $g=0.42)$. Data from the

267 participants' training logs suggested that the two training conditions were equally difficult across

268 the training period. Mean trials over the training period was $28.8(\mathrm{SD}=6.2)$ for the olfactory and

$26927.5(\mathrm{SD}=7.0)$ for the visual training game. This difference was not statistically significant 
$270(\mathrm{t}(70)=0.83, p=0.412$; Hedges's $g=0.20)$. As expected, performance increases on assigned

271 game tasks were observed when comparing pre-training and post-training scores in both the OM

$272(\mathrm{t}(40)=6.02, p<0.001$, Cohen's $d=0.94)$, and the VM group $(\mathrm{t}(30)=10.80, p<0.001$, Cohen's

$273 d=1.94)$. However, the magnitude of the pre-post performance increases for the assigned game

274 was not significantly different across training groups $(\mathrm{t}(70)=-1.69, p=0.095$, Hedges's $g=$ -

275 0.38). On average, neither of the training groups approached the ceiling level of the "virtual 276 experts" (mean trials $=18.9 ; \mathrm{SD}=0.83 ;$ Fig. 2$)$.

278 The results of a two-factor mixed-design ANOVA showed significant interaction effects between 279 time (pre- vs. post-training) and training modality (odor vs. visual) for both the $\mathrm{OM}(\mathrm{F}(1,40)=$ $\left.28023.8, p<0.001, \eta_{\mathrm{p}}{ }^{2}=0.254\right)$ and the $\operatorname{VM}$ task $\left(\mathrm{F}(1,40)=7.71, p=0.007, \eta_{\mathrm{p}}{ }^{2}=0.099\right)$. These 281 within-subject effects indicate that as expected, the largest performance increases were observed 282 in the game tasks that the participants trained.

284 To test whether the training regimes differed in associated performance gains, we first 285 investigated between-subject effects of training modality (odor vs. visual) and time (pre- vs. 286 post-training) on the OM and VM task performances, separately. Results for the OM task yielded 287 significant interaction effects of time and training modality $\left(\mathrm{F}(1,40)=9.59, p=0.003, \eta_{\mathrm{p}}{ }^{2}=\right.$ $2880.12)$ but this was not the case for the $\operatorname{VM}$ task $\left(\mathrm{F}(1,40)=0.44, p=0.510, \eta_{\mathrm{p}}{ }^{2}=0.006\right)$, showing 289 that performance gains were significantly different across groups for the OM game while 290 performance gains were not significantly different across groups for the VM game. The results 291 indicate that olfactory training led to a performance increase in both the olfactory and the visual 292 game, while visual training only led to a performance increase in the visual game. 

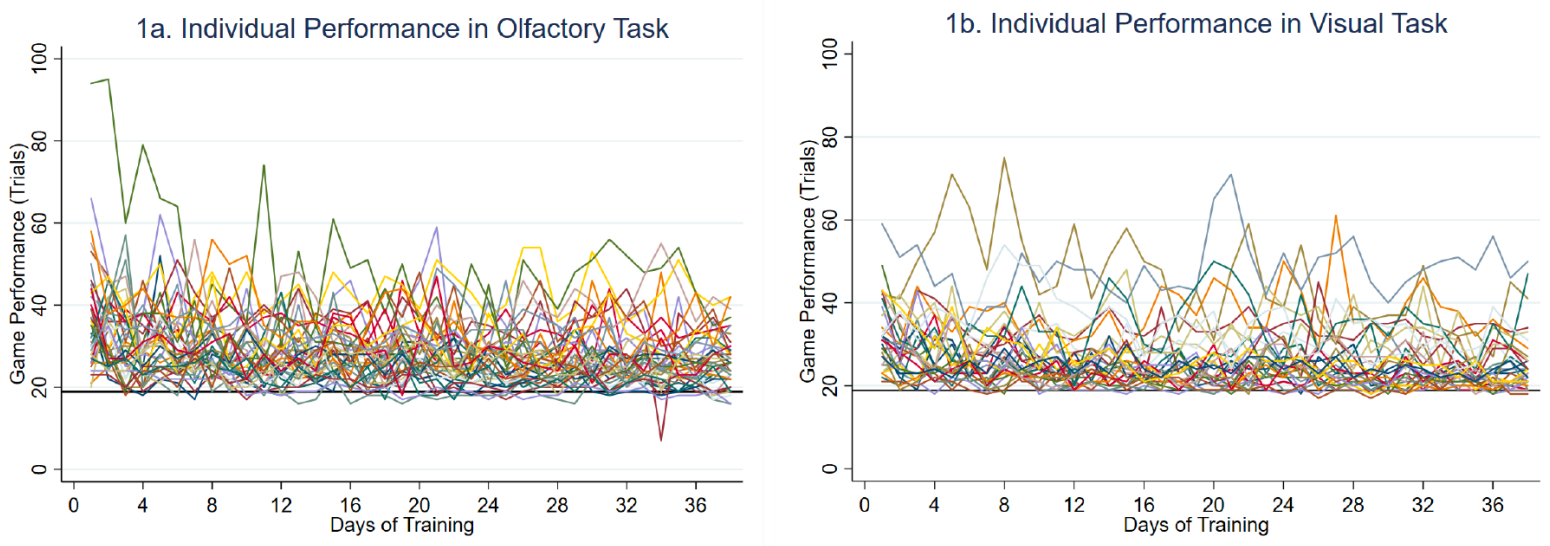

Figure 1 (A-B). Panel plots of individual game performance measured as trials needed to complete the memory task

297 during the training period for the olfactory training group (A) and visual training group (B). Ceiling level was established by data-simulated "virtual experts" (black solid line).
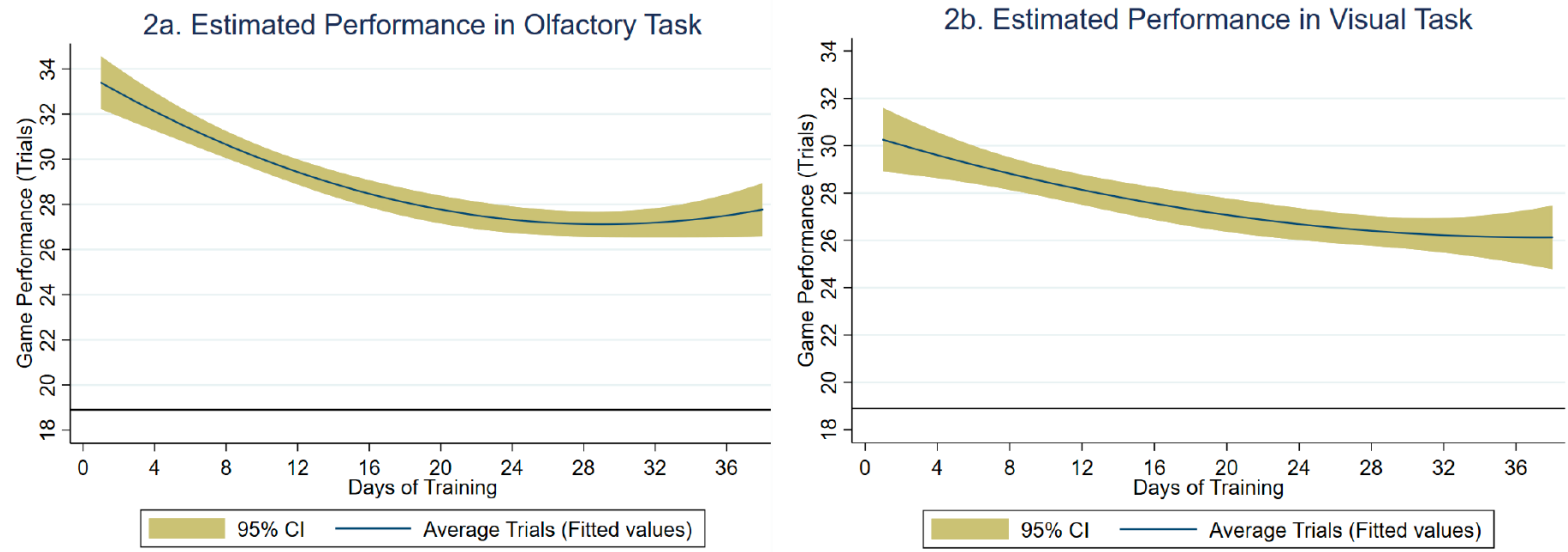

302 Figure 2 (A-B). Average change (blue line) in task performance (trials) estimated as a quadratic function of days of

303 training for the olfactory training group (A) and visual training group (B) with 95\% CIs. Ceiling level was

304 established by data-simulated "virtual experts" (black solid line). 
306 Indeed, post hoc comparisons with t-tests showed that OM training resulted in improved VM

307 performance $(\mathrm{t}(40)=4.42, \mathrm{p}<0.001$, Cohen's $d=0.69)$, but VM training did not result in

308 improved OM performance $(\mathrm{t}(30)=0.97, \mathrm{p}=0.339$, Cohen's $d=0.17)$, suggesting that transfer

309 was asymmetrical. Of key importance for our main a priori hypothesis, transfer gain for the OM

310 group in the visual memory task was larger than the corresponding transfer gain for the VM

311 group in the odor memory task, $(\mathrm{t}(70)=2.444, \mathrm{p}<0.017$, Hedges's $g=0.65$; Fig. 3A-B). These

312 analyses showed that for the OM training group, the transfer gain on the visual memory task was

313 in fact similar to the training gain in the odor task. Results thus indicate a one-sided transfer of

314 learning from the OM to the VM task.

315
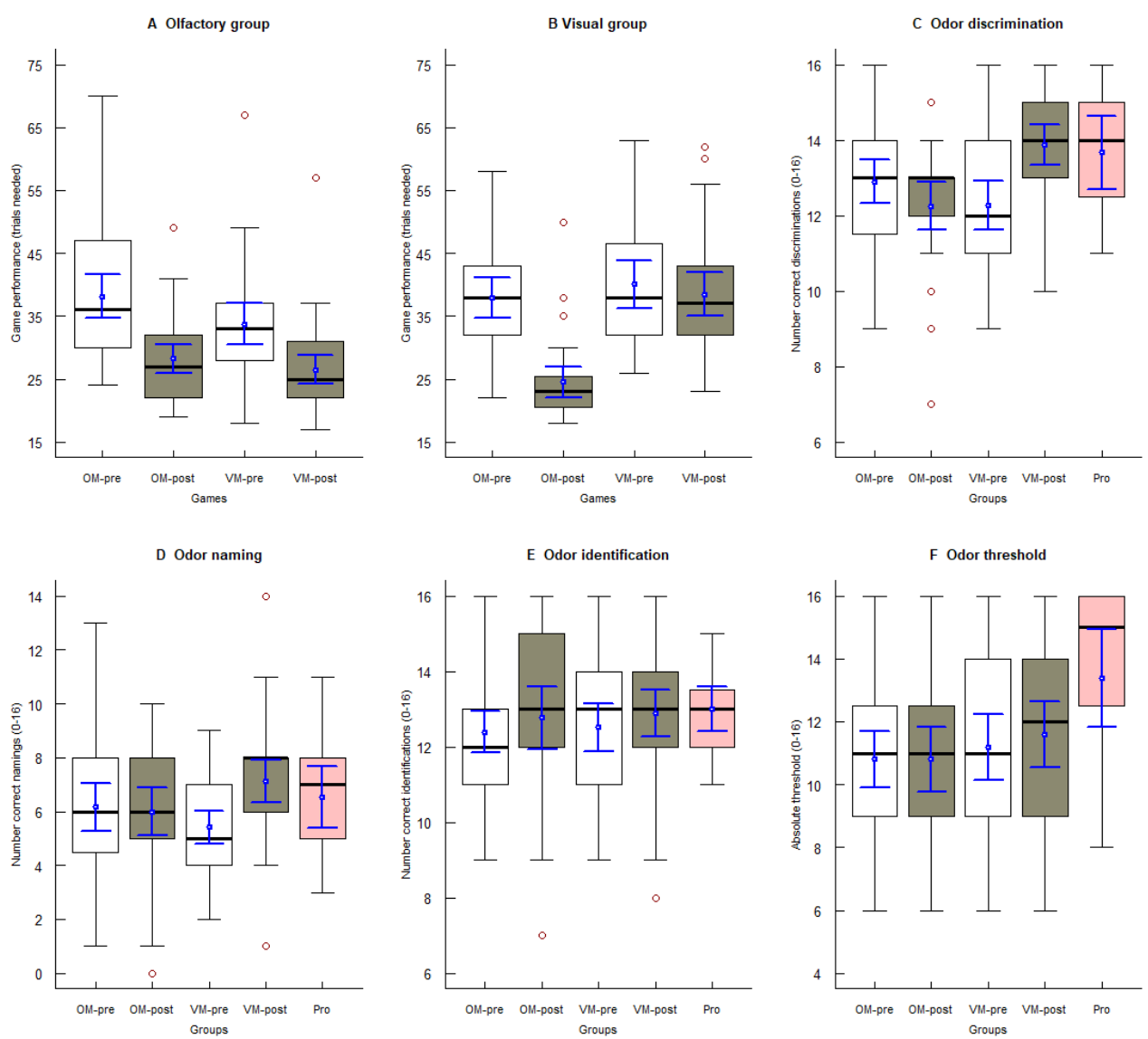
317 Figure 3 (A - B) Boxplots of performance (trials needed) on olfactory memory (OM) and visual memory (VM)

318 tasks at pre-training (white boxes) and post-training (grey boxes) for the two training groups, and score on the

319 olfactory control tasks, (C) odor discrimination, (D) odor naming, (E) odor identification, and (F) butanol odor

320 threshold. Results are shown for the visual memory training group and olfactory memory training group and for

321 olfactory professionals (Pro; pink boxes). Boxplots are displayed separately for pre-training (white boxes) and post-

322 training (grey boxes), with the exception of professionals who did not participate in training or post-training

323 assessment. The boxes indicate the 25, 50 (median), and 75 percentiles of the distribution (lower, middle, and upper

324 horizontal lines of the box). The upper hinges indicate the maximum value of the variable located within a distance

325 of 1.5 times the inter-quartile range above the 75 -percentile. The lower hinges indicate the corresponding distance to

326 the 25 -percentile value. Circles indicate values outside these hinges (outliers). The means and 95\% confidence

327 intervals (dots and error bars in blue) are superimposed on the boxplots.

Olfactory baseline performance. Independent samples t-tests showed that baseline performance

330 levels were similar for the two training groups in all olfactory tasks ( $p$ s $>0.173$, Hedges's $g<$

3310.32 ) and memory games ( $p$ s $>0.077$; Fig. 3). Baseline performances on the olfactory control

332 tasks in the OM group were not significantly correlated with baseline OM game score ( $p$ s >

3330.251 , Hedges's $g<0.42$ ). The results suggest that the two groups were well matched, and that

334 initial OM game performance did not depend on varying olfactory acuity.

336 Follow-up analyses in the OM group revealed no relationship between baseline performances on

337 the olfactory tasks and training-related increase in the OM game $(p s>0.247)$, suggesting that

338 OM gains did not depend on baseline olfactory acuity. Transfer from olfactory training to the

339 VM game were also not associated with baseline olfactory test scores ( $p$ s $>0.116)$. 
341 Comparison to wine professionals. When comparing wine professionals to the pre-training OM

342 group, wine professionals performed better on the odor threshold $(\mathrm{t}(54)=2.285, p=0.026$,

343 Hedges's $g=0.68)$ and odor discrimination tasks $(\mathrm{t}(54)=2.320, p=0.02$, Hedges's $g=0.69)$ but

344 not on the odor identification $(\mathrm{t}(54)=0.905, p=0.369$, Hedges's $g=0.27)$ or odor naming tasks

$345(\mathrm{t}(54)=0.1 .864, p=0.068$, Hedges's $g=0.55)$, although the latter was close to the significance

346 threshold. Following OM training, however, performance was similar to the professionals in all

347 olfactory tasks, including odor naming $(\mathrm{t}(54)=0.819, p=0.416$, Hedges's $\mathrm{g}=0.24)$ and odor

348 discrimination $(\mathrm{t}(54)=0.407, p=0.685$, Hedges's $g=0.12)$, odor threshold $(\mathrm{t}(54)=1.878, p=$

3490.066 , Hedges's $g=0.56)$, and odor identification $(\mathrm{t}(54)=0.182, p=0.856$, Hedges's $g=0.05)$.

350 In contrast, and as expected, the VM group post-training still performed more poorly compared

351 to the professionals on odor threshold $(\mathrm{t}(44)=2.881, p=0.006$, Hedges's $g=0.89)$ and odor

352 discrimination $(\mathrm{t}(44)=2.536, p=0.015$, Hedges's $g=0.78)$, but not odor identification $(\mathrm{t}(44)=$

$3530.0362, p=0.719$; Hedges's $g=0.11)$ or odor naming $(\mathrm{t}(44)=0.723, p=0.473$, Hedges's $g=$

$3540.22)$.

356 Discussion

357 Olfaction is well integrated with the neural systems supporting memory encoding, but the role of

358 smell-based interventions to enhance memory functions is unexplored. Our main result show that

359 engaging the olfactory system in a memory training task was associated with a transfer effect to a

360 similar visual memory task, as well as to untrained olfactory tasks. In contrast, participants who

361 trained with the visual memory task did not show any improvements on the olfactory memory

362 task. The transfer effects were observed in olfactory training, but not in visual training, even

363 though task difficulty and learning rates were comparable for the two training tasks. Based on 
364 our results, we speculate that memory training that engages the olfactory system might promote

365 cross-sensory transfer to a larger extent than is the case for the visual system (which is the

366 dominant model for cognitive interventions). Our results also emphasize that training-related

367 transfer is often unrelated to the magnitude of gains in the trained task (Bjork, 2018).

368

369 Further investigations are needed before definitive conclusions can be drawn about the

370 usefulness of olfactory-based cognitive training. It is unclear if it was the multisensory nature of

371 the memory tasks, or unknown differences in cognitive demands, rather than the engagement of

372 olfaction per se, that produced transfer. While this interpretation cannot be ruled out in the

373 present data and should be the focus of further experiments, it should be noted that each training

374 task used one type of sensory stimulus, visual objects and odors, respectively, that was absent in

375 the other. Thus, we view the sensory complexity as comparable across the two conditions, but to

376 conclusively establish this notion, further experimentation is needed where multisensory

377 complexity of the training tasks is manipulated.

379 Assuming that olfaction indeed promotes cross-sensory cognitive transfer, the mechanisms are

380 unknown, but previous research in visual cognitive training provide clues that help us speculate

381 about its cause. In visual working memory training, the overlapping neural substrates of the

382 trained and transfer tasks is a key determinant of transfer (Dahlin et al., 2008). Thus, the present

383 observation of "asymmetric transfer", from olfactory memory training to visual memory gain,

384 but not vice versa, might indicate that olfactory input provides a relatively unmediated input to

385 the mediotemporal memory encoding regions (Zelano et al., 2016), whereas the visual system

386 involves extensive prior processing in many intermediate cortical regions (Felleman \& Van 
387 Essen, 1991). From the perspective that these additional processing stages act as information

388 filters, visual processing networks will generate a sparse output (Solomon \& Pelli, 1994). By this

389 logic, we speculate that the relatively unfiltered olfactory input to the memory encoding regions

390 (see e.g. Olofsson \& Gottfried, 2015) might potentiate transfer effects due to an increased

391 overlap with memory encoding neural networks. We acknowledge that future studies are needed

392 in order to clarify the behavioral and neuronal mechanisms that might explain transfer from

393 olfactory to non-olfactory tasks. Future studies should also investigate whether these effects

394 replicate and generalize to other forms of olfactory-based cognitive training. Of particular

395 interest is the development of digital olfactory technologies that enable online monitoring of

396 olfactory performance and adjusting difficulty levels along with performance gains, etc (see e.g.

397 Niedenthal et al., 2019).

399 In this work, we satisfied several key criteria that are rarely met in cognitive intervention studies

400 (Simons et al., 2016). First, we established a very high level of similarity between the task format

401 of our interventions, as they differed only in the sensory channels that were stimulated, and not

402 the spatial memory format. Second, we were able to monitor performance during training, a rare

403 feature in olfactory-based interventions (Pekala et al., 2016). Our data from the training period

404 ruled out the possibility that trivial retest effects could account for our enhanced learning of the

405 visual task in the OM group; indeed, day-by-day learning rates were on average very small and

406 could not generate strong test-retest effects. Third, we compared results with those obtained from

407 both "virtual experts" with optimal performance (in game tasks), and wine professionals (in

408 olfactory perception tasks), to establish points of reference for training. These features helped

409 support our interpretation that olfactory-based memory training produces substantial transfer 
410 effects. Future work is needed to elucidate whether odor-based memory transfer effects might be

411 sustained for several months, as are reported in some visual training studies (Constantinidis \&

412 Klingberg, 2016; Sandberg \& Stigsdotter Neely, 2016). Future work should also address how

413 olfactory memory training might produce transfer effects onto untrained tasks that shares neither

414 the sensory modality, nor the task format of the training task (i.e. "far transfer"). Given the

415 present results, odor-based cognitive training might be expected to produce enhanced transfer

416 compared to visual-based training.

418 We hope that the results of our study will stimulate further research on odor-based cognitive

419 interventions. Such interventions could be useful in older individuals, since olfactory

420 impairments constitute early markers for age-related cognitive impairment and dementia

421 (Devanand et al., 2015; Olofsson et al., 2009; Stanciu et al., 2014). It remains to be seen whether

422 odor learning interventions will permit transfer to non-olfactory cognition in older individuals,

423 but such studies are warranted given the devastating impact of memory loss and dementia in the

424 aging population (Wimo et al., 2013).

425

426 Conflict of Interest: None declared.

427 Funding: This study was supported by the Swedish Foundation for Humanities and Social

428 Sciences (grant number M14-0375:1) to ML; Swedish Research Council (grant number 421-

429 2012-806); Marianne and Marcus Wallenberg Foundation (grant number 2014:0178); Knut and

430 Alice Wallenberg Foundation (grant number 2016:0229) to JKO. 
432 Acknowledgments: The authors thank Helene Ålund, Marie Nord, Jasenko Dervisic, Timo

433 Mäntylä, Anders Sand, and our colleagues at the Stockholm University Department of

434 Psychology for valuable assistance and consultation.

435 
436 437

\section{Supplementary materials.}
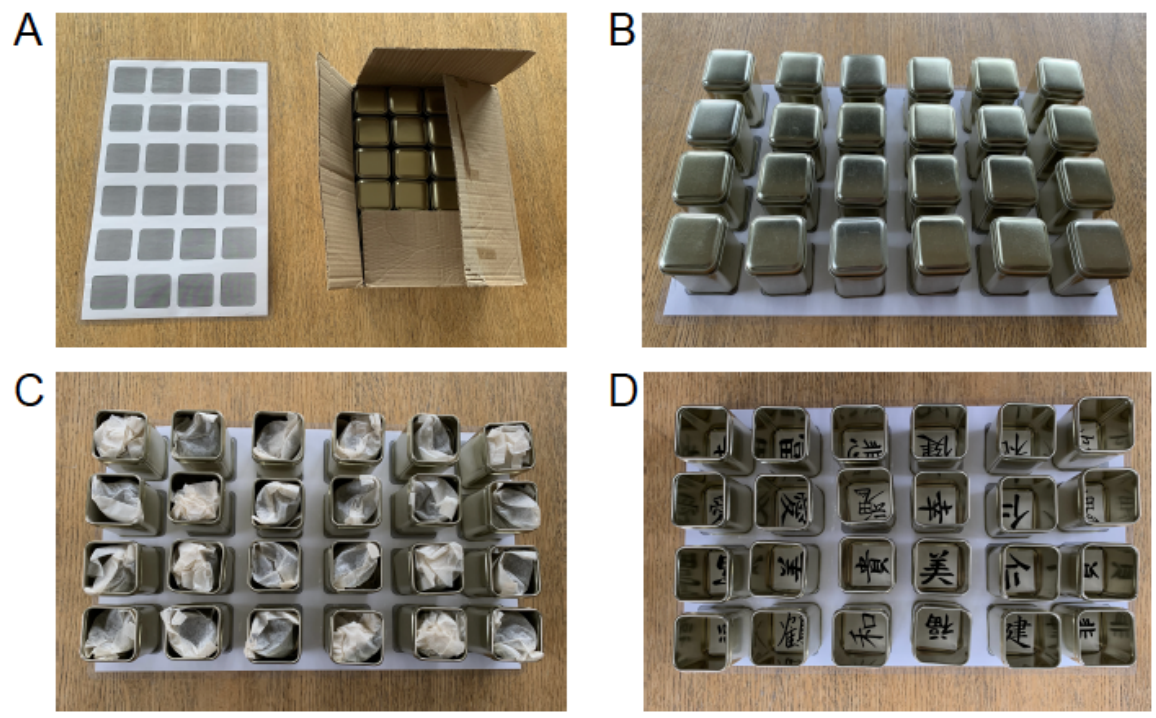

E
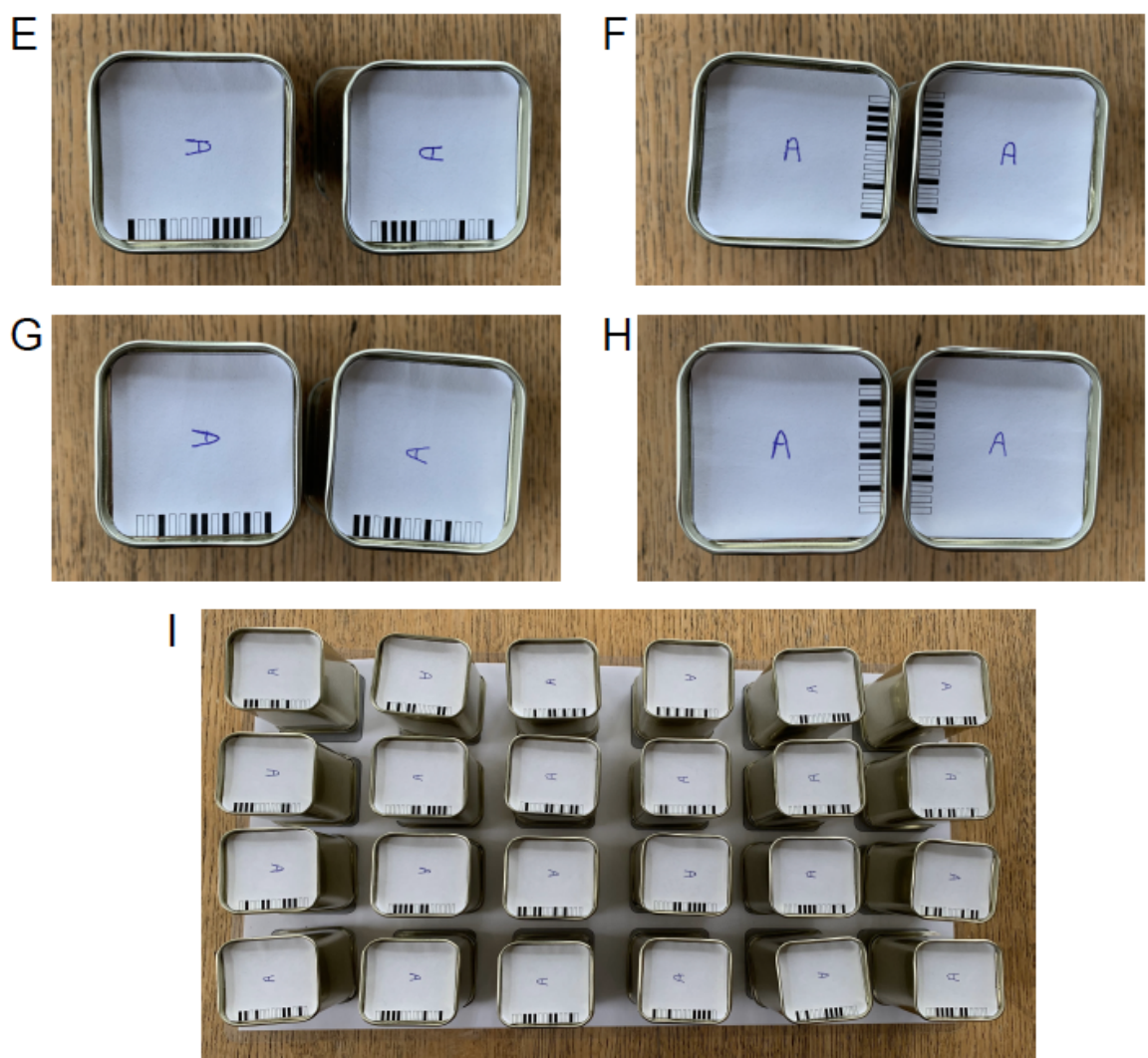

Supplementary Figure 1 (A-I). Memory training equipment. A. Participants were provided with a game board, showing the placement of the cans, and two parallel sets of either olfactory or visual memory games (one of which is shown inside its cardboard box). Participants were also provided with a booklet with detailed instructions for daily training and a booklet where they logged their performance on a trial-by-trial basis (in Swedish, not shown here) B. The placement of the cans during training onset. C. Open cans in the olfactory task. Each can includes a tea bag 
446 with content for odor-based training. Participants only opened two cans for sampling on each 447 trial, so this display serves only to illustrate the content of the cans in the olfactory task. D. Open 448 cans in the visual task. Each can includes a Mandarin Chinese or Korean language symbol for 449 visual-based training. Participants only opened two cans for sampling on each trial, so this 450 display serves only to illustrate the content of the can in the visual task. E. Example of barcodes 451 that are located under the cans and used for confirming whether two sampled cans are matching 452 or not. Due to the mirror-reversed design of the barcodes for matching cans, each of the 24 cans 453 has an unique barcode pattern when viewed individually (irrespectively of whether they are 454 matching or not). F. Example of barcodes for a matching pair. When facing each other, barcodes 455 align, confirming that the stimuli are matching. Upon confirming a match with barcodes, the 456 cans are removed from the board. G. Example of barcodes for a non-matching pair. When 457 viewed individually, it is difficult to imagine what the matching barcode would look like (as 458 there are 12 pairs per set and no individual bar code is identical to another). H. When facing each 459 other, barcodes do not align, confirming the stimluli are not matching. The cans are returned to 460 the board. I. Overview of all barcodes (cans turned upside down and aligned). Participants are 461 instructed to only view barcodes when encountering a perceived match. This ensures minimal 462 exposure to barcodes during training, and as the cans are removed when a match is encountered, 463 the barcodes are not useful as memory cues. 
466 Supplementary Table 1. Demographic and performance data on Sniffin' Sticks assessments for 467 wine professionals.

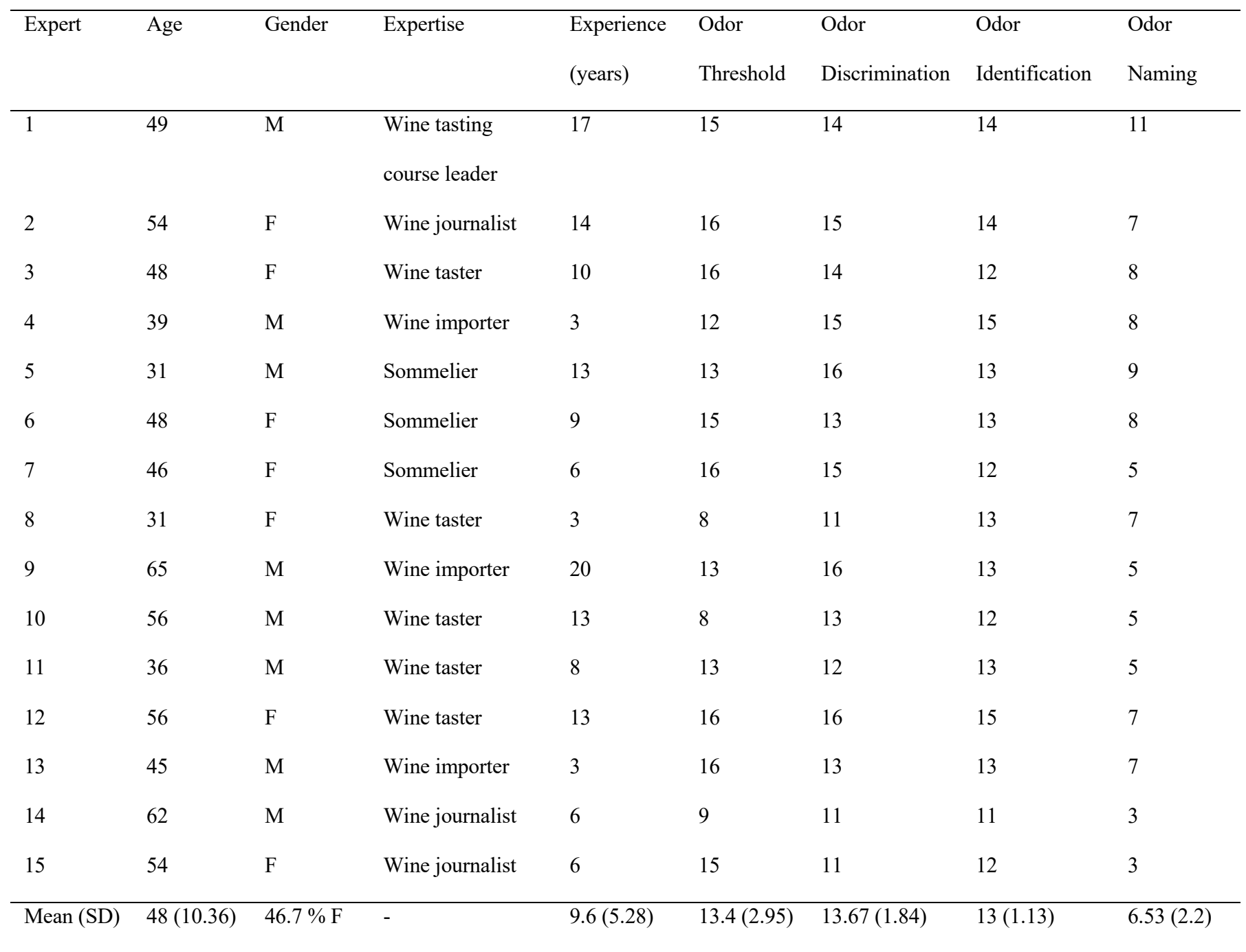

$468 \mathrm{M}=$ Male; $\mathrm{F}=$ Female; $\mathrm{SD}=$ Standard Deviation.

469

470

471

472 


\section{References}

Altundag, A., Cayonu, M., Kayabasoglu, G., Salihoglu, M., Tekeli, H., Saglam, O., \& Hummel, T. (2015). Modified olfactory training in patients with postinfectious olfactory loss. The Laryngoscope, 125(8), 1763-1766. https://doi.org/10.1002/lary.25245 Bergman-Nutley, S., \& Klingberg, T. (2014). Effect of working memory training on working memory, arithmetic and following instructions. Psychological Research, 78(6), 869-877. https://doi.org/10.1007/s00426-014-0614-0 A Journal of the Association for Psychological Science, 13(2), 146-148. https://doi.org/10.1177/1745691617690642

Buschkuehl, M., Hernandez-Garcia, L., Jaeggi, S. M., Bernard, J. A., \& Jonides, J. (2014). Neural effects of short-term training on working memory. Cognitive, Affective \& Behavioral Neuroscience, 14(1), 147-160. https://doi.org/10.3758/s13415-013-0244-9

Constantinidis, C., \& Klingberg, T. (2016). The neuroscience of working memory capacity and training. Nature Reviews. Neuroscience, 17(7), 438-449. https://doi.org/10.1038/nrn.2016.43 Dahlin, E., Neely, A. S., Larsson, A., Bäckman, L., \& Nyberg, L. (2008). Transfer of learning after updating training mediated by the striatum. Science (New York, N.Y.), 320(5882), 15101512. https://doi.org/10.1126/science.1155466

Damm, M., Pikart, L. K., Reimann, H., Burkert, S., Göktas, Ö., Haxel, B., Frey, S., Charalampakis, I., Beule, A., Renner, B., Hummel, T., \& Hüttenbrink, K.-B. (2014). Olfactory training is helpful in postinfectious olfactory loss: a randomized, controlled, multicenter study. The Laryngoscope, 124(4), 826-831. https://doi.org/10.1002/lary.24340

Delon-Martin, C., Plailly, J., Fonlupt, P., Veyrac, A., \& Royet, J.-P. (2013). Perfumers' expertise induces structural reorganization in olfactory brain regions. NeuroImage, 68, 55-62. https://doi.org/10.1016/j.neuroimage.2012.11.044

Devanand, D. P., Lee, S., Manly, J., Andrews, H., Schupf, N., Doty, R. L., Stern, Y., Zahodne, L. B., Louis, E. D., \& Mayeux, R. (2015). Olfactory deficits predict cognitive decline and Alzheimer dementia in an urban community. Neurology, 84(2), 182-189. https://doi.org/10.1212/WNL.0000000000001132 Felleman, D. J., \& Van Essen, D. C. (1991). Distributed hierarchical processing in the primate cerebral cortex. Cerebral Cortex (New York, N.Y.: 1991), 1(1), 1-47. https://doi.org/10.1093/cercor/1.1.1 Fletcher, M. L. (2012). Olfactory aversive conditioning alters olfactory bulb mitral/tufted cell glomerular odor responses. Frontiers in Systems Neuroscience, 6, 16. https://doi.org/10.3389/fnsys.2012.00016 Hummel, T., Kobal, G., Gudziol, H., \& Mackay-Sim, A. (2007). Normative data for the "Sniffin" Sticks" including tests of odor identification, odor discrimination, and olfactory thresholds: an upgrade based on a group of more than 3,000 subjects." European Archives of Oto-Rhino-Laryngology, 264(3), 237-243. https://doi.org/10.1007/s00405-006-0173-0 Hummel, T., Sekinger, B., Wolf, S. R., Pauli, E., \& Kobal, G. (1997). "Sniffin” sticks': olfactory performance assessed by the combined testing of odor identification, odor discrimination and olfactory threshold. Chemical Senses, 22(1), 39-52. 
Hummel, Thomas, Rissom, K., Reden, J., Hähner, A., Weidenbecher, M., \& Hüttenbrink, K.-B. (2009). Effects of olfactory training in patients with olfactory loss. The Laryngoscope, 119(3), 496-499. https://doi.org/10.1002/lary.20101

Kass, M. D., Rosenthal, M. C., Pottackal, J., \& McGann, J. P. (2013). Fear learning enhances neural responses to threat-predictive sensory stimuli. Science (New York, N.Y.), 342(6164), 1389-1392. https://doi.org/10.1126/science.1244916

Kobal, G., Hummel, T., Sekinger, B., Barz, S., Roscher, S., \& Wolf, S. (1996). "Sniffin”' sticks": screening of olfactory performance." Rhinology, 34(4), 222-226.

Kunz, L., Schröder, T. N., Lee, H., Montag, C., Lachmann, B., Sariyska, R., Reuter, M., Stirnberg, R., Stöcker, T., Messing-Floeter, P. C., Fell, J., Doeller, C. F., \& Axmacher, N. (2015). Reduced grid-cell-like representations in adults at genetic risk for Alzheimer's disease. Science (New York, N.Y.), 350(6259), 430-433. https://doi.org/10.1126/science.aac8128 Li, W., Howard, J. D., Parrish, T. B., \& Gottfried, J. A. (2008). Aversive learning enhances perceptual and cortical discrimination of indiscriminable odor cues. Science, 319(5871), 18421845. https://doi.org/10.1126/science.1152837 Lindenberger, U., Wenger, E., \& Lövdén, M. (2017). Towards a stronger science of human plasticity. Nature Reviews Neuroscience, 18(5), 261-262. https://doi.org/10.1038/nrn.2017.44 Mainland, J. D., Bremner, E. A., Young, N., Johnson, B. N., Khan, R. M., Bensafi, M., \& Sobel, N. (2002). Olfactory plasticity: one nostril knows what the other learns. Nature, 419(6909), 802. https://doi.org/10.1038/419802a

Mahncke, H. W., Connor, B. B., Appelman, J., Ahsanuddin, O. N., Hardy, J. L., Wood, R. A., Joyce, N. M., Boniske, T., Atkins, S. M., \& Merzenich, M. M. (2006). Memory enhancement in healthy older adults using a brain plasticity-based training program: a randomized, controlled study. Proceedings of the National Academy of Sciences of the United States of America, 103(33), 12523-12528. https://doi.org/10.1073/pnas.0605194103

Melby-Lervåg, M., Redick, T. S., \& Hulme, C. (2016). Working Memory Training Does Not Improve Performance on Measures of Intelligence or Other Measures of "Far Transfer": Evidence From a Meta-Analytic Review. Perspectives on Psychological Science: A Journal of the Association for Psychological Science, 11(4), 512-534.

https://doi.org/10.1177/1745691616635612

Morquecho-Campos, P., Larsson, M., Boesveldt, S., \& Olofsson, J. K. (2019). Achieving Olfactory Expertise: Training for Transfer in Odor Identification. Chemical Senses, 44(3), 197203. https://doi.org/10.1093/chemse/bjz007

Niedenthal, S., Lundén, P., Ehrndal, M., \& Olofsson, J. K. (2019) A Handheld Olfactory Display For Smell-Enabled VR Games, 2019 IEEE International Symposium on Olfaction and Electronic Nose (ISOEN), Fukuoka, Japan, 1-4. https://doi.org/10.1109/ISOEN.2019.8823162.

Olofsson, J. K., \& Gottfried, J. A. (2015). The muted sense: neurocognitive limitations of olfactory language. Trends in Cognitive Sciences, 19(6), 314-321. https://doi.org/10.1016/j.tics.2015.04.007

Olofsson, J. K., Niedenthal, S., Ehrndal, M., Zakrzewska, M., Wartel, A., \& Larsson, M. (2017). Beyond Smell-O-Vision: Possibilities for Smell-Based Digital Media. Simulation \& Gaming, 48(4), 455-479. https://doi.org/10.1177/1046878117702184

Olofsson, J. K., Rogalski, E., Harrison, T., Mesulam, M.-M., Gottfried, J. A. (2013). A cortical pathway to olfactory naming: evidence from primary progressive aphasia, Brain, 136(4), 12451259. https://doi.org/10.1093/brain/awt019

Olofsson, J. K., Rönnlund, M., Nordin, S., Nyberg, L., Nilsson, L.-G., \& Larsson, M. (2009). 
Odor identification deficit as a predictor of five-year global cognitive change: interactive effects with age and ApoE-epsilon4. Behavior Genetics, 39(5), 496-503. https://doi.org/10.1007/s10519-009-9289-5 Owen, A. M., Hampshire, A., Grahn, J. A., Stenton, R., Dajani, S., Burns, A. S., Howard, R. J., \& Ballard, C. G. (2010). Putting brain training to the test. Nature, 465(7299), 775-778. https://doi.org/10.1038/nature09042

Pekala, K., Chandra, R. K., \& Turner, J. H. (2016). Efficacy of olfactory training in patients with olfactory loss: a systematic review and meta-analysis. International Forum of Allergy \& Rhinology, 6(3), 299-307. https://doi.org/10.1002/alr.21669

Rasch, B., Büchel, C., Gais, S., \& Born, J. (2007). Odor cues during slow-wave sleep prompt declarative memory consolidation. Science (New York, N.Y.), 315(5817), 1426-1429. https://doi.org/10.1126/science.1138581 Royet, J.-P., Plailly, J., Saive, A.-L., Veyrac, A., \& Delon-Martin, C. (2013). The impact of expertise in olfaction. Frontiers in Psychology, 4, 928. https://doi.org/10.3389/fpsyg.2013.00928 Sandberg, P., \& Stigsdotter Neely, A. (2016). Long-term effects of executive process training in young and old adults. Neuropsychological Rehabilitation, 26(5-6), 761-782.

https://doi.org/10.1080/09602011.2015.1108205

Schneiders, J. A., Opitz, B., Krick, C. M., \& Mecklinger, A. (2011). Separating intra-modal and across-modal training effects in visual working memory: an fMRI investigation. Cerebral Cortex (New York, N.Y.: 1991), 21(11), 2555-2564. https://doi.org/10.1093/cercor/bhr037

Simons, D. J., Boot, W. R., Charness, N., Gathercole, S. E., Chabris, C. F., Hambrick, D. Z., \& Stine-Morrow, E. A. L. (2016). Do "Brain-Training" Programs Work? Psychological Science in the Public Interest, 17(3), 103-186. https://doi.org/10.1177/1529100616661983 Solomon, J. A., \& Pelli, D. G. (1994). The visual filter mediating letter identification. Nature, 369(6479), 395-397. https://doi.org/10.1038/369395a0

Stanciu, I., Larsson, M., Nordin, S., Adolfsson, R., Nilsson, L.-G., \& Olofsson, J. K. (2014). Olfactory impairment and subjective olfactory complaints independently predict conversion to dementia: a longitudinal, population-based study. Journal of the International Neuropsychological Society, 20(2), 209-217. https://doi.org/10.1017/S1355617713001409 Teixeira-Santos, A. C., Moreira, C. S., Magalhães, R., Magalhães, C., Pereira, D. R., Leite, J., Carvalho, S., \& Sampaio, A. (2019). Reviewing working memory training gains in healthy older adults: A meta-analytic review of transfer for cognitive outcomes. Neuroscience and Biobehavioral Reviews, 103, 163-177. https://doi.org/10.1016/j.neubiorev.2019.05.009 van Rijzingen, I. M., Gispen, W. H., \& Spruijt, B. M. (1995). Olfactory bulbectomy temporarily impairs Morris maze performance: an $\mathrm{ACTH}(4-9)$ analog accellerates return of function. Physiology \& Behavior, 58(1), 147-152.

Wimo, A., Jönsson, L., Bond, J., Prince, M., Winblad, B., \& Alzheimer Disease International. (2013). The worldwide economic impact of dementia 2010. Alzheimer's \& Dementia: The Journal of the Alzheimer's Association, 9(1), 1-11.e3. https://doi.org/10.1016/j.jalz.2012.11.006 Zelano, C., Jiang, H., Zhou, G., Arora, N., Schuele, S., Rosenow, J., \& Gottfried, J. A. (2016). Nasal Respiration Entrains Human Limbic Oscillations and Modulates Cognitive Function. The Journal of Neuroscience, 36(49), 12448-12467. https://doi.org/10.1523/JNEUROSCI.258616.2016

Zelcer, I., Cohen, H., Richter-Levin, G., Lebiosn, T., Grossberger, T., \& Barkai, E. (2006). A cellular correlate of learning-induced metaplasticity in the hippocampus. Cerebral Cortex (New York, N.Y.: 1991), 16(4), 460-468. https://doi.org/10.1093/cercor/bhi125 
609 Zelinski, E. M. (2009). Far transfer in cognitive training of older adults. Restorative Neurology 610 and Neuroscience, 27(5), 455-471. https://doi.org/10.3233/RNN-2009-0495

611

612 